Mathematics requires to be digested and assimilated as well as acquired. The trend of our work is to cover as many subjects and as much ground as possible, and this continual advance with its successive generalizations and its revelations of incompleteness in what has been accepted as complete tends to cultivate a lack of confidence, accentuated if the student is embarrassed by difficulties of detail. This feeling of uncertainty takes shape in reliance upon others (book or person) to pronounce final judgment upon whatever is done. Perhaps something could be done to give the student the more secure grip on the secondary school subjects which the teacher must have by taking up these subjects again at the close of the college course and treating their principles and methods (not new details, as a rule), with the greater thoroughness and breadth of view which are made possible by the collegiate mathematics as outlined in the minimum course above.

VI. Difference between the work of the prospective teacher and the prospective graduate student of mathematics. - In the college this difference may be very slight.

VII. The teachers needed (in college) to carry out the above programme fully must have the wide horizon of a university training.

The Woman's College,

W. H. Maltbie.

BALTIMORE, MD.

\title{
ON A MEMOIR BY RICCARDO DE PAOLIS.
}

BY PROFESSOR CHARLOTTE ANGAS SCOTT.

ABour twenty years ago de Paolis published a series of memoirs* dealing with the $(2,1)$ transformation of the plane; of these the second and third are concerned with applications of the theory to non-euclidean geometry and to

* "Le trasformazioni piane doppie" ; Atti d. r. Accad.d. Lincei, series $3^{\text {a }}$, vol. 1 (1877); pp. 511-544.

"La trasformazione piana doppia di secondo ordine, e la sua applicazione alla geometria non euclidea" ; Atti d. r. Accad. d. Lincei, series $3^{\mathrm{a}}$, vol. 2 (1878); pp. 31-50.

"La trasformazione piana doppia di terzo ordine, primo genere, e la sua applicazione alle curve del quarto ordine" ; Atti d. r. Accad. d. Lincei, series $3^{\mathrm{a}}$, vol. 2 (1878); pp. 851-878. 
quartic curves, while the first treats of the general theory of the $(2,1)$ transformation. De Paolis employs no analysis, all his results are obtained simply by geometrical considerations. The curves of the first plane (piano semplice) which correspond to the straight lines of the second plane (piano doppio) have only two free intersections, all the other common points being absorbed by the fixed (or fundamental) points by means of which the conditions to which the curves are subject are imposed. This, taking up a question which arises in connection with a memoir by Clebsch, $*$ is the next in order to Cremona's memoirs, $†$ the $(2,1)$ transformation naturally following the $(1,1)$. This very striking memoir, being already classical, is a fair subject for discussion; and in the following pages I propose to consider, $\$$ in particular, de Paolis's classification of fundamental points and lines, which in my opinion he has based on symptoms of differences instead of on the real causes. When the $(2,1)$ transformation is regarded as but one case in the general theory of $(x, 1)$ transformation, some of the special properties-those, in fact, to which its peculiar interest is largely due-take on a rather different aspect.

The relation connecting the dimensions $k$ of a system of curves of genus $p$ with the number $x$ of variable intersections, is known to be

$$
x-(k-1)=p,
$$

if the fundamental points are entirely arbitrary in position, and consequently impose independent conditions ;

$$
x-(k-1) \leq p-1,
$$

if the points are connected in such a way that the conditions imposed are not all independent. This shows that for the $(2,1)$ transformation with arbitrary fundamental points,

$$
p+k=3 \text {. }
$$

Hence, as shown by de Paolis, there are two cases to consider, namely,

$$
p=1, k=2 ; \quad p=0, k=3 .
$$

\footnotetext{
* Clebsch, "Ueber den Zusammenhang einer Classe von Flächenabbildungen mit der Zweitheilung der Abel': chen Functionen" ; Math. Annalen, vol. 3 (1871).

†Cremona, "Sulle trasformazioni geometriche delle figure piane" ; Memorie d. Accad. d. Bologna, series $2^{\mathrm{a}}$, vol. 2 (1863) ; vol. 5 (1865).

$\ddagger$ I have not attempted to keep to the lettering of the memoir, preferring rather to use that adopted in other places.
} 
In the first of these the transformation net is composed of all curves that satisfy the assigned conditions; in the second, these curves form a three-fold infinity, from which the net is selected by means of three determining curves. Now so far as the determination by fundamental points is concerned, these three curves may be any three independent ones of the system that have not any additional points in common. The same thing holds whatever value $x$ may have; the system of curves determined by the fundamental points is of dimensions $k$, and to obtain a transformation net we virtually impose $k-2$ extrinsic conditions, these being implied in the choice of the three determining curves. The theory of linear systems contained in a system of the same order, but a greater number of dimensions, has received very little special attention, owing to the fact that the theorems relating to such a system are simply deducible from those for the complete system; but there are one or two points in the theory that deserve to be remarked, and they are the ones that seem to me to throw some light on the true nature of the differences that de Paolis has attempted to formulate in dividing fundamental points, as also fundamental curves, into three species.

Let the curves, $\varphi$, of the first plane that correspond to the straight lines of the second plane be given as of order $\sigma$, having fixed multiple points of orders $\rho_{1}, \rho_{2}, \ldots$, at the fundamental points. There may be also fundamental curves. A curve $\beta$, of order $\omega$, with multiple points of orders $\tau_{1}, \tau_{2} \ldots$ at the fundamental points, is called fundamental if it meets the curves of the given system only at the fixed points, that is, if

$$
\sum \rho_{i} \tau_{i}=\sigma \omega .
$$

These curves have been minutely studied by various Italian writers, * who have shown that any such curve is in general rational, and precisely determined by the fundamental points through which it passes, facts which lead to the equations

$$
\sum \tau_{i}^{2}=\omega^{2}+1, \quad \sum \tau_{i}=3 \omega-1 \quad \text { (Bertini). }
$$

* Caporali, "Sopra i sistemi lineari triplamente infiniti di curve algebriche piane" ; Collect. Mat. In Mem. Dom. Chelini, 1879.

Jung, "Sui sistemi lineari di curve algebriche di genere qualunque" ; Rend. del $R$. Ist. Lomb., series $2^{\mathrm{a}}$, vol. 20 (1887).

Bertini, "Sulle curve fondamentali dei sistemi lineari di curve piane algebriche" ; Rend. del Circ. Mat. di Palermo, vol. 3 (1889).

Castelnuovo, "Ricerche generali sopra i sistemi lineari di curve piane" ; Mem. della R. Accad. di Sc. di Torino, series $2^{\mathrm{a}}$, vol. 42 (1891). 
If however the fundamental points are specialized in such a manner that the conditions actually imposed on the curves $\varphi$ are fewer, by $e$, than the conditions apparently imposed (that is, if the fundamental points have an excess $*$ $e$ for the curves $\varphi$, or if the system of curves $\varphi$ is of sovrabbondanza $\dagger e$ ), then $\beta$ need not be rational ; its genus may have any value that does not exceed $e$ (Castelnuovo). Any irreducible fundamental curve $\beta$ is a factor in $k$ independent curves of the system, that is, in a contained $(k-1)$-fold system ; and there is the general theorem that $\beta$ will be a non-repeated factor in the generic curve of this $(k-1)$-fold system.

As regards the fundamental points and curves, two questions call for investigation. The first relates to the possibility of a curve with a multiple point of order higher than the stated value $\rho$ at a fundamental point, or, we may say, to the existence of a $\varphi$ that shall be irregular at one or more of the fundamental points ; the second to the possibility of a curve with $\beta$ as a repeated factor, without any specialization in the position of the fundamental points. These two questions I have considered in another place; $\ddagger$ hence the results only need be mentioned here.

On the first question the conclusion is that the $k$-fold system of curves contains a curve that is irregular at any fundamental point whose order is less than $k$ (or, by an extension of the argument, at any $m$ fundamental points the sum of whose orders does not exceed $k-m$ ); but in selecting from the complete system three curves for the determination of a net, no two must be chosen that are irregular at the same point, for such a choice would introduce new fundamental points indefinitely near to the stated ones. Irregularity on one curve at any fundamental point does not of itself imply any specialization in the fundamental points.

As to the second question, the result is that the $k+1$ determining curves of the system can be chosen as the first $k+1$ of the following series:-

one, that does not contain $\beta$,

two, that contain $\beta$ but neither contain nor produce $\beta^{2}$ (i.e. when taken in any linear combination),

* Macaulay, "Point groups in relation to curves," Proc. Lond. Math. Soc., vol. 26 (1895).

† Castelnuovo, loc. cit.

+ "Note on linear systems of curves," Nieuw Archief voor Wiskunde, Tweede Reeks, Derde Deel, 1898 ; also Bulletin, vol. 4 (1897-8); p. 88. 
three, that contain $\beta^{2}$, but neither contain nor produce $\beta^{3}$,

$h+1$, that contain $\beta^{h}$, but neither contain nor produce $\beta^{h+1}$, where every $\rho \geqq h$ times the corresponding $\tau$, and at least one $\rho<(h+1)$ times the corresponding $\tau$.

If however $k+1>\frac{1}{2}(h+1)(h+2)$, there are also curves, $k+1-\frac{1}{2}(h+1)(h+2)$ in number, containing higher powers of $\beta$; the numbers containing successive powers being $N_{h+1}, N_{h+2}$, etc., no general formula for an $N$ can be given, all that can be said is that the successive $N$ 's do not increase. As these last contain $\beta$ to a power so high that $h \tau>\rho$ at some one point, the curves are irregular. Hence it is convenient to distinguish the two parts of the series as regular and irregular. It is at once evident that any one of the curves can be modified by the addition of multiples of later members of the series without affecting its relation to $\beta$.

If $k=2$, the curves by which the net is determined are necessarily the first three, that is,

$$
\varphi_{1}, \quad \beta \psi_{2}, \quad \beta \psi_{3} .
$$

If $k>2$, any three may be chosen that have not any fixed points in common other than those expressly provided for at the fundamental points. One must obviously be $\varphi_{1}$; of the other two, one may belong to the irregular part of the series, but not both, inasmuch as this would cause irregularity at one point on both curves.

For example, the 12-fold system of 15-ics, with multiple points of orders $8,6,6,5,4,4,4$ has the cubic $2,1,1,1,1$, 1,1 as a fundamental curve; and $h=4$. The complete system can therefore be written as follows, where all thirteen curves are regular :

$\varphi_{1}, \quad \beta\left(\psi_{1}, \psi_{2}\right), \quad \beta^{2}\left(\chi_{1}, \chi_{2}, \chi_{3}\right), \quad \beta^{3}\left(\vartheta_{1}, \vartheta_{2}, \vartheta_{3}, \vartheta_{4}\right), \quad \beta^{4}\left(\zeta_{1}, \zeta_{2}, \zeta_{3}\right)$.

The system residual to $\beta^{4}$ is the net of cubics with fixed points of orders $0,2,2,1,0,0,0$, which is simply the straight line $A_{2} A_{3} \times$ the net of conics through $A_{2} A_{3} A_{4}$. Hence in this case we must not choose, for the determination of a net, two curves containing $\beta^{4}$, even though both would be regular, for the net thus determined would have other fixed points, namely, the three points in which $A_{2} A_{3}$ meets the 15-ic $\varphi_{1}$. But with the 12-fold system of 14-ics with points of order $8,6,4,4,4,4,4$, which has the same fundamental curve, there is not this restriction on freedom of choice. 
We can at once write down a system with any possible fundamental curve, and with any arbitrary residual system, by means of the theorem :-If a curve $\beta$ of order $\omega$, rational and precisely determined by fixed points of order $\tau_{1}, \tau_{2}, \cdots$, which impose independent conditions, has $h$ free intersections with the generic curve of a $q$-fold system of $n$-ics conditioned solely by its behavior at the fixed points, having these as multiple points of orders $r_{1}, r_{2}, \cdots$, then $\beta$ is a fundamental curve of the system of $(n+h \omega)$-ics determined by multiple points of orders $r_{1}+h \tau_{1}, r_{2}+h \tau_{2}, \cdots$, at the fixed points.

The fundamental curve is not possible unless the numbers expressing the orders of its multiple points satisfy the relations

$$
\begin{aligned}
& \frac{1}{2} \Sigma \tau(\tau+1)=\frac{1}{2} \omega(\omega+3)-\delta, \\
& \frac{1}{2} \Sigma \tau(\tau-1)=\frac{1}{2}(\omega-1)(\omega-2),
\end{aligned}
$$

and then $\delta$ simple points must be taken on $\beta$ to complete its determination.

The proof of the theorem is obvious. The number of free intersections of $\beta$ with the generic curve of the system is

$$
\begin{aligned}
\omega(n+h \omega)- & \Sigma \tau(r+h \tau) \\
& =n \omega-\Sigma r \tau+h\left(\omega^{2}-\Sigma \tau^{2}\right) \\
& =h\left[1+\omega^{2}-\Sigma \tau^{2}\right]=0 .
\end{aligned}
$$

Hence $\beta$ is either an $h$-fold factor or a fundamental curve ; but as the dimensions of the system

$$
\begin{aligned}
& \geq \frac{1}{2}(n+h \omega)(n+h \omega+3)-\frac{1}{2} \Sigma(r+h \tau)(r+1+h \tau) \\
& \geq q+\frac{1}{2} h(h+1)>q,
\end{aligned}
$$

it follows that the system of $(n+h \omega)$-ics is more extensive than the given system of $n$-ics, hence it is not simply this system with an extra factor $\beta^{h}$, but a more extensive system with $\beta$ as a fundamental curve.

By means of this theorem we can write down a system with any desired fundamental curve, choosing a residual system at random. For example, if the fundamental curve be a rational cubic, we must use also for its complete determination six simple points on the curve, which can then be expresssed as $2,1^{6}$. If the residual system is to be all conics, then $h=2 \times 3=6$; hence the desired system consists of 20 -ics $(6 \times 3+2)$, with one multiple point of order 12 , and six sextuple points. 
In the general rational transformation, in which the coördinates of a point in the second plane are given rationally in terms of the coördinates of a point in the first plane by equations of the form

$$
\varphi_{1}: \varphi_{2}: \varphi_{8}=x_{1}^{\prime}: x_{2}^{\prime}: x_{3}^{\prime},
$$

to a single point $P$ in the first plane there corresponds a single point $P^{\prime}$ in the second plane; but to this one point in the second plane there correspond in the first plane all the $x$ points $P_{1}, P_{2}, \cdots, P_{\kappa}$ which are the intersections of the pencil of $\varphi^{\prime} \mathrm{s}$ through $P$. If however $P$ is a fundamental point of order $\rho, e . g .$, the point $(0,0,1)$, there is no longer any unique correspondent $P^{\prime}$. The $\varphi$ 's being arranged by powers of $x_{3}$, the equations become

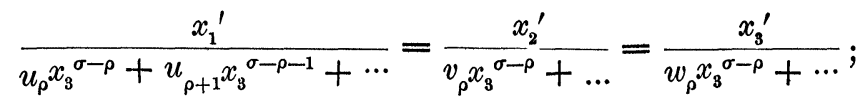

the correspondent to the actual point $(0,0,1)$ is absolutely indeterminate, for all these denominators are zero; but taking a point indefinitely near to $(0,0,1)$, in a definite direction given by $x_{1}=\lambda x_{2}$, the corresponding point is determinate, the equations now becoming

$$
\frac{x_{1}^{\prime}}{u_{\rho}(\lambda, 1)}=\frac{x_{2}^{\prime}}{v_{\rho}(\lambda, 1)}=\frac{x_{3}{ }^{\prime}}{w_{\rho}(\lambda, 1)} \text {. }
$$

Taking in succession points close to $(0,0,1)$ in all directions, that is, allowing $P$ to describe an indefinitely small simple circuit about $(0,0,1), P^{\prime}$ describes a curve $\alpha^{\prime}$, rational and of order $\rho$, inasmuch as the $x_{1}{ }^{\prime}, x_{2}{ }^{\prime}, x_{3}{ }^{\prime}$ are rationally expressed in terms of the parameter $\lambda$ by these equations. (See Clebsch-Lindemann, and other places.) Returning to the first plane, the complete correspondent to $\alpha_{i}^{\prime}$ is a curve $a_{i}$ of order $\sigma \rho_{i}$, which can be shown* to have at $A_{i}$ a multiple point of order $\rho_{i} \rho_{j}$, but at $A_{i}$ one of order $\rho_{i}{ }^{{ }^{3}}+1$. This curve $a_{i}$ is the complement of the fundamental point, the locus of the $x-1$ points associated with a point that describes a circuit about $A_{i}$. If $\rho_{i}=1$, it is simply the particular $\varphi$ that has a double point at $A_{i}$.

Thus while any ordinary point of the first plane corresponds to a point of the second plane, and is associated with $x-1$ points of the first plane, there are exceptional points, namely, the fundamental points ; any one of these in

*C. A. Scott, "Studies in the transformation of plane algebraic curves"; Quar. Jour. of Math., vol. 29 (1898). 
general corresponds to a curve, and is associated with a curve which replaces the $x-1$ points.

If however one $\varphi, \varphi_{3}$ suppose, be irregular at $(0,0,1)$, the lowest terms being of degree $\rho^{\prime}>\rho$, the equations are

$$
\frac{x_{1}^{\prime}}{u_{\rho}+\cdots}=\frac{x_{2}^{\prime}}{v_{\rho}+\cdots}=\frac{x_{3}{ }^{\prime}}{w_{\rho^{\prime}}+\cdots} \text {. }
$$

As $x_{1}, x_{2}$ are indefinitely small, and $\rho^{\prime}>\rho$, to a point in a definite direction from $(0,0,1)$ there corresponds a definite point on $x_{8}^{\prime}=0$; and as the complete correspondent to this is $\varphi_{3}=0$, the curve complementary to $A_{i}$ is $\varphi_{3}$. For consistency of algebraic statement, it appears that the fundamental curve in the second plane must be taken as $x_{3}{ }^{\prime \prime}$, and the curve complementary to $A_{i}$ as $\varphi_{3}{ }^{p}$.

If $\varphi_{3}$ is irregular at another fundamental point of stated order $\rho_{k}$, the correspondent to this is the same line $x_{3}{ }^{\prime}$ taken $\rho_{k}$ times, and the complement is the same $\varphi_{3}$ taken $\rho_{k}$ times.

The existence of the irregular $\varphi$ affects the position of the points associated with any point $P$ in the immediate neighborhood of $A_{i}$. These are to be determined as the free intersections of two $\varphi$ 's through $P$; two such $\varphi$ 's have in general $\rho^{2}$ fixed intersections at $A_{i}$, but if one of the two has a $\rho^{\prime}$-point, the number is $\rho \rho^{\prime}$; hence of the usually free intersections $\rho\left(\rho^{\prime}-\rho\right)$ lie at $A_{i}$ instead of only one. This number, $\rho\left(\rho^{\prime}-\rho\right)$, is greater than unity unless $\rho=1, \rho^{\prime}=2$. Now the raising of a simple point to a double point involves the imposition of two conditions only, hence is always possible in a net; it seems better therefore not to regard this case as an irregularity, seeing that it is the necessary occurrence.

If moreover $\varphi_{3}$ is irregular also at $A_{j}$, then, taking $P$ close to $A_{i}$ as before, instead of the usual $\rho_{j}^{2}$ intersections at $A_{j}$ there are $\rho_{j} \rho_{j}^{\prime}$; thus of the $*$ points of the group, $\rho_{j}\left(\rho_{j}^{\prime}-\rho_{j}\right)^{\prime}$ lie at $A_{j^{*}}$ It is immaterial in what direction $P$ lies from $A_{i}$, for an irregular $\varphi$ belongs to the pencil of $\varphi$ 's determined by any direction whatever.

Hence irregularity on a $\varphi$ will interfere with the customary association of a fundamental point with a curve; the fundamental point may have complementary points lying at other fundamental points, or even coinciding with itself. In the cases treated by de Paolis, since $x=2$ there is only one point associated with $A_{i}$, and this point

(i) describes a curve; $A_{i}$ is then a fundamental point of the first species ;

(ii) lies at another fundamental point $A_{j} ; A_{i}$ is then a fundamental point of the second species; 
(iii) lies at $A_{i}$, which is then a fundamental point of the third species. Such a point arises, as has just been shown, from irregularity on one $\varphi$; it can arise also from another cause, the presence of what de Paolis calls a double point, to be explained later.

For a non-specialized transformation system with $x=2$, $p+k=3$. If $p=1, k=2$; the only "irregularity" possible on $a$ is the existence of a double point where the stated conditions impose only a simple point, and it has already been pointed out that this cannot properly be regarded as an irregularity. If $p=0, k=3$; we cannot produce irregularity on one $\varphi$ at two points, for this requires the imposition of four conditions, and is therefore possible in a system only if the generic curve has as many as four degrees of freedom. Hence the fundamental points of the second species do not present themselves unless the determining points of the system are specialized, in which case $p+k>3$. The fundamental points of the third species can occur, for $k=3$ allows us to raise a double point to a triple point, and then an extra intersection lies at $A_{i}, A_{i}$ is associated with itself ; there is no complementary curve, for the two points of a group are fully accounted for.

This classification of fundamental points lends itself to the case $x=2$, the characteristics of the three species being then mutually exclusive, inasmuch as the one point associated with any point $P$, near to $A_{i}$. cannot satisfy more than one of the three conditions. But if $x>2, e . g .,=4$, and $k$ is sufficiently great, we may have the three points complementary to $P$ exhibiting all three characteristics. As $P$ describes an indefinitely small simple circuit about $A_{i}, P_{1}$ may describe a curve, $P_{2}$ may lie at $A_{j}, P_{3}$ at $A_{i}$, and then the curve described by $P_{1}$ will be simply the irregular $\varphi, \varphi_{3}$. Thus de Paolis' classification is based on properties which are insufficient for purposes of discrimination when $x>2$.

Two points of a general $x$ group (the intersections of a pencil of $\varphi$ 's), can come together only if the $\varphi$ 's have contact; the locus of such points is therefore the Jacobian of $\varphi_{1}, \varphi_{2}, \varphi_{3}$. On forming this, in certain circumstances factors will present themselves, possibly to be rejected in finding the locus of points of true contact. De Paolis calls this locus the "curva doppia"; it is the Jacobian reduced by the rejection of irrelevant factors. As a point $P_{1}$, together with its associate $P_{2}$, describes the Jacobian, the correspondent $P^{\prime}$ describes a curve in the second plane, the "curva limita" of de Paolis [synoptic curve, Q. J., loc. cit.]. In the general theory, with $x>2$, we have to take into ac- 
count also the co-jacobian, the locus of the remaining points $P_{3}, P_{4}, \cdots, P_{\kappa}$; a detailed study of the relations of these curves, and their use in transformation, is to be found in the article in the Quarterly Journal already referred to.

Examining the equations of the Jacobian, it is seen to represent a curve of order $3(\sigma-1)$, with multiple points of order $3 \rho-1$. If however one $\varphi$ is irregular at $A_{i}$, having there a multiple point of order $\rho^{\prime}$, the order of this point on the Jacobian is $\rho^{\prime}+2 \rho-2$; the terms of this order are affected with a numerical factor $\rho^{\prime}-\rho$. If now $\rho^{\prime}=\rho+1$, this has the normal value $3 \rho-1$; but if $\rho^{\prime} \geq \rho+2$, then $\rho^{\prime}+2 \rho-2 \geq 3 \rho$. Thus the Jacobian is not affected by the simplest type of irregularity, that in which the order of a fundamental $\rho$-point is augmented by unity on one $\varphi$. With the restriction on the scope of the investigation adopted by de Paolis, namely, $x=2$, it is not possible to have $\rho^{\prime}=$ $\rho+2$, hence this irregularity on the Jacobian is not mentioned. The irregularity that is considered on p. 523 has a different origin ; it is due to the presence of a double point, whose nature is considered separately.

Corresponding to the fundamental points in the first plane there are fundamental curves in the second plane, which de Paolis classes as of the first, second, or third kind, according to the nature of the fundamental points from which they are derived. He makes the statement, as regards those of the second and third kind, that they meet the curves of the second plane which correspond to the straight lines of the first plane in fixed points only. This is not the case if $x>2$; but possibly it is true for $x=2$, as stated by de Paolis, and not only for the curves that correspond to straight lines, but also for the correspondents to any curves in the first plane, though I must confess I find de Paolis' argument somewhat obscure.

If there be in the first plane a fundamental curve $\beta$, the equations of transformation are

$$
\varphi_{1}: \beta^{h_{2}} \psi_{2}: \beta^{h_{3}} \psi_{3}=x_{1}^{\prime}: x_{2}^{\prime}: x_{3}^{\prime} .
$$

Corresponding to any point on $\beta$ we have in the second plane the point $B^{\prime}(1,0,0)$; to the point $B^{\prime}$ there correspond all the intersections of $\beta^{h_{2}} \psi_{2}, \beta^{h_{3}} \psi_{3}$ that do not lie at the fundamental points, $x-h_{2} h_{3}$ in number. Hence the fundamental curve $\beta$ replaces $h_{2} h_{3}$ of a group of $x$ points, thus displaying another exception to the general laws of the transformation. Of course $h_{2} h_{3}$ is necessarily less than $x$. If $h_{2}=h_{3}=h$, to any point in the immediate neighbor- 
hood of $B^{\prime}$, in the direction $x_{2}{ }^{\prime}=\lambda x_{3}{ }^{\prime}$, there correspond $h$ points on $\beta$, inasmuch as $\beta=0$ meets $\psi_{2}-\lambda \psi_{3}^{\prime}=0$ in precisely $h$ points ; thus if $h=1$, that is, if $\beta$ is a fundamental curve of the simplest type, as a point $P^{\prime}$ describes an indefinitely small circuit about $B^{\prime}$ one of the corresponding points describes the curve $\beta$; the relation of $B^{\prime}$ to $\beta$ is precisely analogous to the relation of $A$ to $\alpha^{\prime}$. This however does not hold if $h$ is greater than unity.

If $h_{2}<h_{3}$, to any point in the neighborhood of $B^{\prime}$ there correspond $h_{2}$ fixed points on $\beta$, determined by $\psi_{2}=0$.

Thus if $h_{2} h_{3}>1$, one or more of the correspondents to $\beta$ is $\beta$ itself, the remainder being a. set of $x-h_{2} h_{3}$ points.

If however $\psi_{2}, \psi_{3}$ have a common factor $\gamma$, we have in the simplest case the equations

$$
\varphi_{1}: \beta \gamma \vartheta_{2}: \beta \gamma \vartheta_{3}=x_{1}^{\prime}: x_{2}^{\prime}: x_{3}^{\prime} \text {. }
$$

To the point $B^{\prime}$ there now corresponds the reducible curve $\beta \gamma$, which is "monovalent" as regards the system of $\varphi$ 's; that is to say, if the $\varphi$ is made to pass through one point on either component, it contains $\beta_{\gamma}$ as a factor, the whole curve $\beta \gamma$ is equivalent to a single point as means of imposing conditions on the generic curve of the system of ' $\varphi$ 's. (Castelnuovo, loc. cit.) With any point on $\beta$ there are associated the $x-2$ intersections $*$ of $\vartheta_{2}, \vartheta_{3}$, and the curve $\gamma$; this curve is therefore a part of the complement of $\beta$.

If finally the equations of transformation are

$$
\varphi_{1}: \beta^{h_{2}} \gamma^{k_{2}} \vartheta_{2}: \beta^{h_{3}} \gamma^{k_{3}} \vartheta_{3}=x_{1}{ }^{\prime}: x_{2}{ }^{\prime}: x_{3}{ }^{\prime},
$$

then a group of $x$ associated elements, of which $\beta$ forms a part, contains in the simplest case $x-h_{2} h_{3}-k_{2} k_{3}$ points, not on $\beta \gamma$, together with $\beta, h_{2} h_{3}$ times, and $\gamma, k_{2} k_{3}$ times. If for instance $x=4$, and the transformation net is

$$
\varphi_{1}, \quad \beta \gamma \vartheta_{2}, \quad \beta^{2} \gamma^{2} \vartheta_{3},
$$

then a set of four associated elements one of which is $\beta$ consists of $\beta$, twice, $\gamma$, once, and one point. De Paolis divides the fundamental curves in the first plane into three kinds, according as the single associate (the only one if $x=2$ ) is a point, another curve, or the curve itself; but here again, just as in the case of the fundamental points, if $x>2$ the characteristics may coexist in any combination, hence the classification cannot profitably be placed on this basis in general.

* There are $\kappa-2$ in the simplest case that can arise, but they may be fewer in number. 
On p. 518 de Paolis asserts that while fundamental curves of the first and second species are rational, those of the third species are hyperelliptic. Surely he has here been misled by the $(2,1)$ correspondence of the points of such a a curve with directions proceeding from $B^{\prime}$. Any fundamental curve $\beta$, of whatever species, is subject to the conditions formulated in the memoirs of Bertini and Castelnuovo, conditions which are a direct consequence of the fact that all the intersections of $\beta$ with the generic $\varphi$ are fixed. If the complete system from which the $\varphi$-net is derived by selection has no "sovrabbondanza," then $\beta$ is rational, since. its genus cannot exceed the sovrabbondanza of the system. For a specialized choice of fundamental points, a fundamental curve of any species may cease to be rational.

From the ordinary determinant expression for the Jacobian it is at once seen that a fundamental curve that is an $\left(h_{2}, h_{3}\right)$ factor presents itself as an $\left(h_{2}+h_{3}-1\right)$-fold factor in the Jacobian: rejecting it, the residual part, $J_{0}$, is de Paolis' "curva doppia." This cuts $\beta$ in $h_{2}+h_{3}-2$ points; if $h_{2}=h_{3}=h$, these $2(h-1)$ points are $h-1$ points of simple contact. These are the general facts; de Paolis naturally notices only the cases that can occur for $x=2$. If $\beta$ is a simple fundamental curve, then $\beta$ is a simple factor to be rejected from the Jacobian, and the reduced Jacobian does not meet $\beta$; if however $\beta$ is a fundamental curve of the third species $\left(h_{2}=1, h_{3}=2\right)$, the factor $\beta^{2}$ is to be rejected, and the reduced Jacobian meets $\beta$ in one point.

A point on the "curva doppia" is a point of the plane where two complementary points come together, that is, are consecutive; the $\varphi$ 's through such a point have simple contact, the common tangent being the line determined by these consecutive points, and one $\varphi$ has a double point. If there is an actual coincidence of two complementary points, de Paolis characterizes it as a double point of the plane, stating that the continuous system of points of the first plane may contain a discrete system of points associated with themselves. Any two $\varphi$ 's through such a point have two intersections, not due to contact. If the point is not fundamental, and $x=2$, this can only be by means of a common factor, which is consequently a fundamental curve. The net is then determined by

$$
\varphi_{1}, \quad \beta \psi_{2}, \quad \beta \psi_{3},
$$

but as $\psi_{2}^{\prime}, \psi_{3}^{\prime}$ are to have a common point on $\beta$, which fixes the sole (hitherto) free intersection of $\psi_{2}$ and $\psi_{3}$, it follows 
that $\psi_{3}$ is of the form $\psi_{2}^{\prime}+\beta_{1} \psi_{3}$; hence the net is determined by

$$
\varphi_{1}, \quad \beta \phi_{2}^{\prime}, \quad \beta^{2} \vartheta_{3},
$$

and the fundamental curve $\beta$ is therefore of the third species, as de Paolis shows in a different manner (p. 524). The double point is the one point in which $\beta$ and $\psi_{2}$ intersect ; and as any $\varphi$ that contains $\beta$ is of the form $\beta \psi_{2}^{\prime}+\lambda \beta^{2} \vartheta_{3}$, that is, $\beta\left\{\psi_{2}+\lambda \beta_{1} \psi_{3}\right\}$, every $\operatorname{such} \varphi$ has necessarily a double point at this point.

De Paolis takes the view that all such double points are isolated from the Jacobian, because he found them on the fundamental curves, and these he rejected in forming the "curva doppia." If however $x>2$, all the $\varphi$ 's through a point may have a double point there, without degeneration, that is, without the assistance of fundamental curves; this fact is outside de Paolis' argument, but it is essential to a full understanding. In this case the reduced Jacobian, the " curva doppia," has a double point. Such a point exists, for instance, $*$ at $(1,0,0)$ for the system $\varphi_{1}, \varphi_{2}, \varphi_{3}$, where

$$
\begin{aligned}
& \varphi_{1}=x_{1}\left(x_{1}+x_{2}+x_{3}\right)^{2}, \\
& \varphi_{2}^{\prime}=2 x_{1} x_{2}{ }^{2}+\left(x_{2}-x_{3}\right)\left(x_{2}+x_{3}\right)^{2}, \\
& \varphi_{3}=2 x_{1} x_{3}{ }^{2}-\left(x_{2}-x_{3}\right)\left(x_{2}+x_{3}\right)^{2},
\end{aligned}
$$

whose reduced Jacobian is

that is,

$$
x_{1}\left(x_{2}+x_{3}\right)^{2}-\left(x_{1}+x_{2}+x_{3}\right)\left(x_{2}-x_{3}\right)^{2}=0,
$$

$$
4 x_{1} x_{2} x_{3}-\left(x_{2}+x_{3}\right)\left(x_{2}-x_{3}\right)^{2}=0 .
$$

This point is of the same nature as the double point of which de Paolis speaks, but of higher order.

These are the only two ways in which all $\varphi$ 's of a pencil can have more than one intersection at a point that is not fundamental, independently of contact; either any two, and therefore all, have a common factor ; or any two, and therefore all, have a multiple point.

The question whether a fundamental curve, which is a repeated factor in one or more $\varphi$ 's, shall or shall not be counted as a part of the reduced Jacobian, is after all a mere matter of definition ; we may adopt any convention. A repeated factor ( $h$-fold) in one $\varphi$, that is not a fundamental curve, presents itself as an $(h-1)$-fold factor in the

* Quar. Jour. of Math., vol. 29 (1898), pp. 357 and 379. 
Jacobian, and has to be counted as an integral part of this curve; whereas a simple factor has no special relation to it. This lends some support to the view, to which I incline, that a fundamental curve which occurs as a repeated factor ought to be looked upon as a part of the true Jacobian; and bearing in mind the fact just proved as regards multiple points of the plane, it appears that this convention gives the most consistent set of results.

Again, a double point may be at a fundamental point $A$; a point complementary to $A$ is then at $A$, which is therefore a fundamental point of the third species. This is the second manner of specializing the fundamental point, referred to above. It is the only one that de Paolis considers in any detail; and his purely geometrical treatment, while it has led him to a correct formulation of the properties of such a point, is not very conclusive as to its nature, which however is extremely interesting. This is considered analytically in the Quarterly Journal of Mathematics;* as the results of the investigation throw light on a rather obscure part of the memoir of de Paolis, it appears suitable to give here a résumé.

The pencil formed by the $\varphi$ 's that proceed in any given direction from a fundamental point of order $\rho$, contains one special $\varphi$, with a cuspidal branch whose tangent lies in that direction. If the direction is one of the $3 \rho-1$ indicated by the tangents to the Jacobian, the special $\varphi$ has not a simple cuspidal branch, but a branch with a higher singularity, met by its own tangent in four points. In the simplest case, this can be resolved into two simple branches in contact, and the $\varphi$ might be referred to as tacnodal; but to allow for the more complicated singularity, the term extranodal seems more convenient, as it is applicable to all cases. (Analytically, the repeated factor in the terms of degree $\rho$ is a factor in the terms of degree $\rho+1$.) These extranodal $\varphi$ 's are determined by the $3 \rho-1$ tangents to the Jacobian ; the fundamental point may, however, be specialized in such a manner that for every direction the special $\varphi$ is extranodal instead of cuspidal. This happeus necessarily for all directions if it happens for some one direction in addition to the $3 \rho-1$; and the Jacobian has then a point of order $3 \rho$, while the synoptic curve contains $\alpha^{\prime}$ as a factor. The number of intersections at $A$ of two $\varphi$ 's proceeding in any assigned direction $A P$ is in general $\rho^{2}+1$, but in this case it is $\rho^{2}+2$; of these $\rho^{2}$ are the fixed intersections, which

\footnotetext{
* In the continuation of the article, already referred to, appearing in the current volume of the Quar. Jour. of Math.
} 
are not enumerated in any $x$-group ; thus two points of a $\%$ group fall at $P$, in whatever direction $P$ is taken. This may perhaps be most simply expressed by saying that the complement to $A$ contains an evanescent circuit about $A$. This is what de Paolis refers to as a "punto fondamentale doppio"; it is one variety of the fundamental point of the third species.

The fundamental points and lines, the double points, and the Jacobian, in the first plane, with their correspondents in the second plane, form a kind of framework for the transformation, indicating everything exceptional ; and a complete knowledge of them is essential to a full comprehension of the possible effects of the general rational transformation. If $x>2$, the co-jacobian has to be taken into account also, and as I have here shown, the different species of fundamental points and lines can no longer be discriminated; hence the purely geometrical treatment becomes very involved, and it appears necessary to resort to analysis. Thus it is hardly to be expected that the treatment of transformation can be carried any further on the lines of Cremona and de Paolis; but the intrinsic interest of de Paolis' work is surely excuse enough for devoting some little space to it in a periodical of a critical and historical nature.

BryN MAWR CoLLFGE, May, .900.

\section{NOTES.}

The Chicago Section of the American Mathematical Societr will meet in the Ryerson physical laboratory of the University of Chicago, on Thursday and Friday, December 27 th and 28 th next. Titles and abstracts of papers to be read at this meeting should be in the hands of the Secretary of the Section not later than December 5th.

The third (July) number of the Transactions of the AMerican Mathematical Society contains the following papers : "Wave propagation over non-uniform conductors," by $\mathrm{M}$. I. PuPIN ; "Ueber Systeme von Differentialgleichungen denen vierfach periodische Functionen Genüge leisten," by M. Krause ; "On linear criteria for the determination of the radius of convergence of a power series," by E. B. VAN VLECK ; "On the existence of the Green's function for the 